Short Communication

\title{
Brothers with genu recurvatum
}

Naoto Saitoa,*, Keiji Tensyob, Hiroshi Horiuchib ${ }^{b}$ Kaoru Aokib, Seneki Kobayashi ${ }^{b}$, Hiroyuki Katob, and Tomoki Koshoc

a Department of Applied Physical Therapy, Shinshu University School of Health Science, 3-1-1 Asahi, Matsumoto, Nagano, 390-8621, J apan

bDepartment of Orthopaedic Surgery, Shinshu University School of Medicine, 3-1-1

Asahi, Matsumoto, Nagano, 390-8621, J apan

cDepartment of Medical Genetics, Shinshu University School of Medicine, 3-1-1

Asahi, Matsumoto, Nagano, 390-8621, J apan

* Corresponding author. Department of Applied Physical Therapy, Shinshu University School of Health Science, 3-1-1 Asahi, Matsumoto, Nagano, 390-8621, J apan. Tel.:+81 26337 2409; fax: +81 263372409.

E-mail address: saitoko@hsp.md.shinshu-u.ac.jp (N. Saito).

Keywords: Knee; Genu recurvatum; Deformity; Brothers; Osteoarthritis 


\begin{abstract}
Genu recurvatum deformity is rare, usually resulting from injury to the anterior part of the proximal tibial growth plate. However, a small group of cases have no known cause. We describe brothers with the same type of this deformity on the same side. The degree of deformity differed between them, but deformities began at the same age in both (17 years) and progressed over a short period. There was no history of trauma, or other orthopedic or genetic disorders. These features strongly suggested involvement of heritable risk factors.
\end{abstract}




\section{Case report}

Two brothers 67 and 65 years old consulted our clinic because of pain and genu recurvatum deformity involving the left knee. Neither had any physical problems unrelated to the knee. No general or orthopaedic problems were evident at birth or during childhood development. No known relative had a similar knee deformity.

The older brother developed pain in the left knee with no apparent cause at the age of 17 years. The pain persisted for 1 year, during which it precluded physical activity. The genu recurvatum deformity of the left knee worsened gradually since onset of pain, reaching the present degree of deformity within 1 year. Subsequently the pain disappeared. The patient's activities of daily living were not compromised, and he could participate in sports at the recreational level. Decades later, pain in the left knee became evident on exercise at 55 years, with gradual worsening. Radiographs obtained at 67 years of age showed an osseous recurvatum deformity of 34 degrees affecting the proximal left tibia, and showed devel opment of osteoarthritis (Fig. 1A). Three-dimensional CT images of the left knee showed severe recurvatum deformity (Fig. 2). The patient was treated conservatively, undergoing follow-up on an outpatient basis.

The younger brother developed left knee pain with no known cause when he was 17 years old, the same age of onset as for his older brother. He could not take part in physical activity for about 6 months. Genu recurvatum deformity developed in the left knee to the same degree as at present over the 6 months. The pain subsequently disappeared, and he was able to play recreational sports. Left knee pain became apparent at the age of 58 , while right knee pain developed at almost the same time, with gradual worsening on both sides. On radiographs at 65 years, the proximal left tibia showed osseous recurvatum deformity of 12 degrees (Fig. 1B), while the right knee did not show such a deformity. Both knees showed advanced osteoarthritis. Total knee arthroplasty was performed for both knees, and pain disappeared.

\section{Discussion}

Genu recurvatum deformity, a rare condition with onset in childhood and adolescence, may be caused by bone or soft tissue pathology in the area of the knee. The most common reason for genu recurvatum deformity is fracture, but in other instances minor injury of the proximal tibia may cause premature closure of the anterior part of the proximal tibial growth plate [1-5]. Possible minor injuries are avulsion of the tibial eminence (Osgood-Schlatter disease), skeletal traction by a wire through the proximal tibia, prolonged pressure on the tibial tuberosity by plaster casts or braces, and infectious diseases of the tibia. The brothers reported here had no history of fracture, and they did not have any minor injury of the proximal tibia. Segev et al. reported a case of genu recurvatum possibly due to focal fibrocartilaginous dysplasia of the proximal tibia [2]. This rare lesion usually appears in infants (before the age of 3 years), but the lesions in our cases occurred in adolescence. A few reported occurrences of genu recurvatum deformity in the 
proximal tibia have been considered idiopathic [2,3]. These cases showed no familial pattern of occurrence, and in general no suggestion has been made that this deformity can be related to hereditary factors. The present report of genu recurvatum is the first in which brothers were affected. The degree of deformity differed between them, but deformities began at the same age in both (17 years) and progressed over a short period. These features strongly suggested involvement of heritable risk factors. This condition also may be associated with Blount's disease, which is characterized by varus deformity of the proximal tibia occurring with no evident cause in children and adolescents [6-8]. No genetic tests were performed in these brothers as no target gene has been identified for these conditions. Analysis of more cases will be required to determine causes and possible hereditary background of these rare bone diseases producing deformity of the knee in young persons. 


\section{References}

[1] Moroni A, Pezzuto V, Pompili M, Zinghi G. Proximal osteotomy of the tibia for the treatment of genu recurvatum in adults. J Bone J oint Surg Am 1992;74:577-86.

[2] Segev E, Hendel D, Wientroub S. Genu recurvatum in an adolescent girl: hypothetical etiology and treatment considerations: a case report. J Pediatr Orthop B 2002;11:260-4.

[3] Chen LC, Chan YS, Wang CJ . Opening-wedge osteotomy, allografting with dual buttress plate fixation for severe genu recurvatum caused by partial growth arrest of the proximal tibial physis: a case report. J Orthop Trauma 2004;18:384-7.

[4] Bellicini C, Khoury J G. Correction of genu recurvatum secondary to Osgood-Schlatter disease: a case report. I owa Orthop J 2006;26:130-3.

[5] Domzalski ME, Lipton GE, Lee D, Guille J T. Fractures of the distal tibial metaphysis in children: patterns of injury and results of treatment. J Pediatr Orthop 2006;26:171-6.

[6] Thompson GH, Carter J R. Late-onset tibia vara (Blount's disease): current concepts. Clin Orthop Relat Res 1990;255:24- 35.

[7] Shimode K, Miyagi N, Aoki Y, Yasuda K, Yamazaki S, Minami A. J uvenile Blount's disease: bilateral case with asynchronous onset. J Orthop Sci 2003;8:222-6.

[8] van Huyssteen AL, Hastings CJ , Olesak M, H offman EB. Double-elevating osteotomy for late-presenting infantile Blount's disease: the importance of concomitant lateral epiphysiodesis. J Bone J oint Surg Br 2005;87:710-5. 


\section{Figure legends}

Fig. 1. A: A lateral radiograph of the left knee in the older brother at 67 years. Osseous recurvatum deformity of the proximal tibia measures 34 degrees. B: Genu recurvatum deformity of 12 degrees in the younger brother at 65 years.

Fig. 2. A three-dimensional CT image of the left knee in the older brother shows severe recurvatum deformity. 


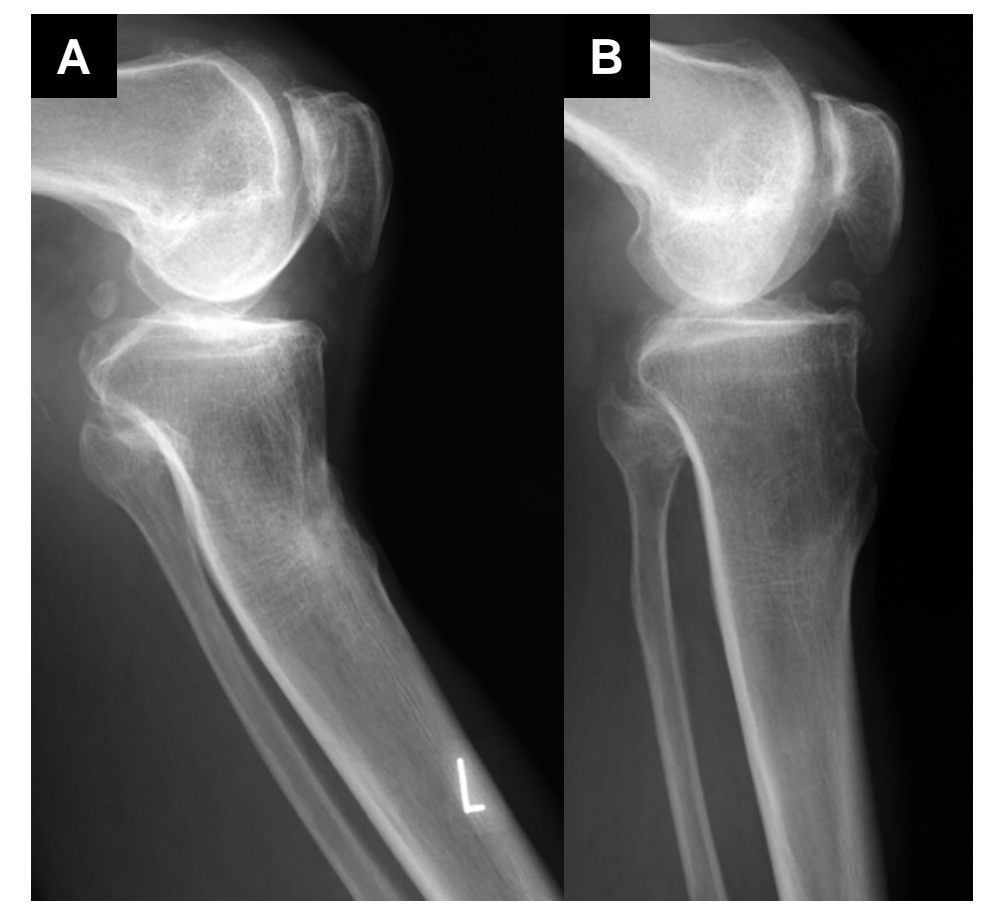

Fig. 1 


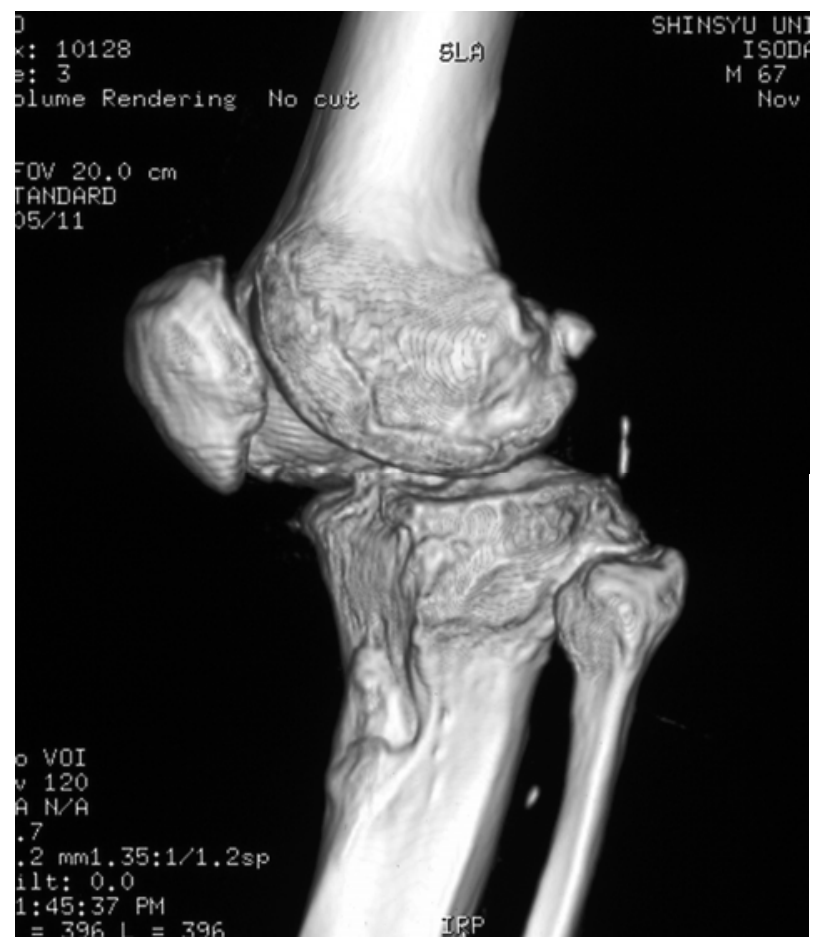

Fig. 2 